\title{
Older women are frailer, but less often die than men: a prospective study of older hospitalized people
}

\author{
Nicola Veronese ${ }^{\mathrm{a}, \mathrm{m}}$, Giacomo Siri ${ }^{\mathrm{a}}$, Alberto Cella ${ }^{\mathrm{a}}$, Julia Daragjati ${ }^{\mathrm{b}}$, Alfonso J. Cruz-Jentoft ${ }^{\mathrm{c}}$, \\ Maria Cristina Polidori ${ }^{\mathrm{d}}$, Francesco Mattace-Raso ${ }^{\mathrm{e}}$, Marc Paccalin ${ }^{\mathrm{f}, \mathrm{g}}$, Eva Topinkova ${ }^{\mathrm{h}}$, \\ Antonio Greco ${ }^{\mathrm{i}}$, Arduino A. Mangoni ${ }^{\mathrm{j}}$, Stefania Maggi ${ }^{\mathrm{k}}$, Luigi Ferrucci ${ }^{1}$, Alberto Pilotto ${ }^{\mathrm{a}, \mathrm{n}, *}$, on \\ behalf of the MPI AGE Investigators
}

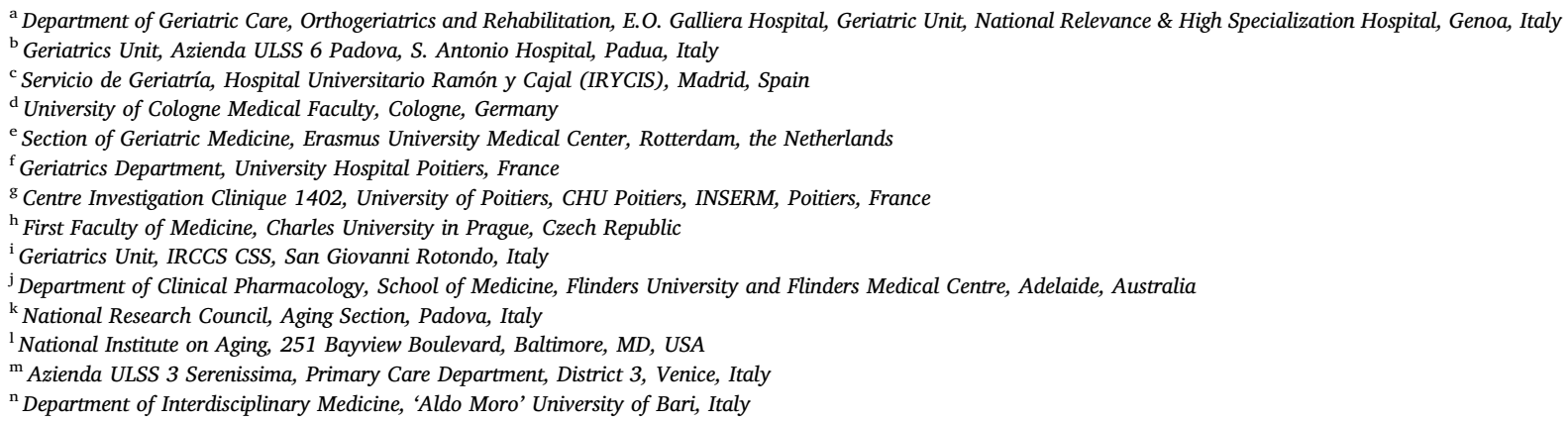

\section{A R T I C L E I N F O}

\section{Keywords:}

Sex

Multidimensional Prognostic Index

Prognosis

Hospital

Cohort study

\begin{abstract}
A B S T R A C T
Objectives: The association between frailty, mortality and sex is complex, but a limited literature is available on this topic, particularly for older hospitalized patients. Therefore, the objective of our study was to prospectively evaluate sex differences in frailty, assessed by the Multidimensional Prognostic Index (MPI) and mortality, institutionalization, and re-hospitalization in an international cohort of older people admitted to hospital. Study design: We used data from nine public hospitals in Europe and Australia, to evaluate sex differences in mortality, frailty and the risk of institutionalization and re-hospitalization, during one year of follow-up. Main outcome measures: People aged 65 years or more admitted to hospital for an acute medical condition or for a relapse of a chronic disease were included. A standardized comprehensive geriatric assessment, which evaluated functional, nutritional, and cognitive status, risk of pressure sores, comorbidities, medications and cohabitation status, was used to calculate the MPI to measure frailty in all hospitalized older people. Data regarding mortality, institutionalization and re-hospitalization were also recorded for one year.

Results: Altogether, 1140 hospitalized patients (mean age $=84.2$ years; 694 women $=60.9 \%$ ) were included. The one-year mortality rate was $33.2 \%$. In multivariate analysis, adjusted for age, MPI score, centre and diagnosis at baseline, although women had higher MPI scores than men, the latter had higher in-hospital (odds ratio, $\mathrm{OR}=2.26 ; 95 \%$ confidence intervals, $\mathrm{CI}=1.27-4.01)$ and one-year post-discharge mortality $(\mathrm{OR}=2.04$; $95 \% \mathrm{CI}=1.50-2.79)$. Furthermore, men were less frequently institutionalized in a care home than female patients $(\mathrm{OR}=0.55$; 95\%CI: $0.34-0.91)$, but they were also more frequently re-hospitalized $(\mathrm{OR}=1.42 ; 95 \% \mathrm{CI}$ : 1.06-1.91) during the year after hospital discharge.

Conclusion: Older hospitalized men were less frail, but experienced higher in-hospital and one-year mortality than women. Women were admitted more frequently to nursing homes and experienced a lower risk of rehospitalization. These findings suggest important differences between the sexes and extends the 'male-female health-survival paradox' to acutely ill patient groups.
\end{abstract}

\footnotetext{
* Corresponding author at: Department of Geriatric Care, Orthogeriatrics and Rehabilitation, E.O. Galliera Hospital, Mura delle Cappuccine 14, 16128 Genoa, Italy.
} E-mail address: alberto.pilotto@galliera.it (A. Pilotto). 


\section{Introduction}

It is widely known that women live longer than men worldwide, regardless of other demographic and clinical characteristics [1]. At the same time, women tend to experience a greater burden of multimorbidity and frailty than men, particularly in older age [2].

Previous systematic reviews showed that the prevalence of frailty was significantly higher in older women than men, both using phenotypic and cumulative deficit models of frailty [3,4]. These findings overall suggest that women have a poorer health status and higher levels of frailty, and a lower risk of mortality, than men [2]. This phenomenon has been defined as the 'male-female health-survival paradox' [5].

Indeed, data on sex differences in frailty and mortality seems to be equivocal. While some studies in older community-dwelling people reported that women showed lower mortality rates than men, after taking in consideration the role of frailty $[6,7]$, other studies reported that frailty is more important, as predictor of mortality, in female than in male subjects [8-11].

Notably, most studies that addressed this question were limited to community dwelling populations. Thus, whether the frailty-mortality paradox also affect older persons who are acutely ill or are hospitalized is unknown. Addressing this question is important because the older population account for $50 \%$ of hospitalized adults [12] despite accounting for only $13 \%$ of the population in the United States, and with the aging of the population this percentage will increase. Moreover, frailty is associated with several negative outcomes in hospitalized older people, including increased length of stay, mortality, institutionalization $[13,14]$. In addition, it has been reported that older women tend to be excluded from certain acute therapeutic interventions more than men, and such selective exclusion may be revealed looking at differential mortality [15].

Finally, to the best of our knowledge, no previous study assessed sex differences in outcomes of public health importance, particularly institutionalization and risk of re-hospitalization, after hospital discharge.

The aim of this study was to prospectively evaluate sex differences in frailty, assessed by the multidimensional prognostic index (MPI) $[16,17]$ and mortality, institutionalization, and re-hospitalization in an international cohort of older people admitted to hospital, during 12 months of follow-up.

\section{Methods}

\subsection{Study population}

This is a secondary analysis of a longitudinal, multicenter, cohort study including 9 Acute Geriatric Units across Europe (Genova, San Giovanni Rotondo and Padova, Italy; Madrid, Spain; Cologne, Germany; Rotterdam, the Netherlands; Poitiers, France; Prague, Czech Republic) and Adelaide, Australia, conducted according to the World Medical Association's 2008 Declaration of Helsinki, the guidelines for Good Clinical Practice, and the Strengthening the Reporting of Observational Studies in Epidemiology (STROBE) guidelines.

Inclusion criteria were: 1 ) age $>65$ years; 2) admitted to hospital for an acute medical condition or for a relapse of a chronic disease; 3 ) ability to provide an informed consent or availability of a proxy for informed consent and willingness to participate in the study; 4) complete CGA during hospitalization. No selective exclusion criteria were used, but 61 patients dropped out at 1-year follow-up. These subjects were excluded from the analysis since we didn't know the censorship date.

Participants were included consecutively in each setting and for participating to the study at least 50 participants should be included. No cut-off was posed for the maximum of participants to be included for each setting. The recruitment period was from February 2015 to July 2016, with the end of one-year follow-up in August 2017.
The study was initially approved by the Ethics Committee of the leading center (Padova) and the documentation was sent and approved by the other centers. The Ethical Committees of each center then approved this observational study. Informed consent was given by participants who underwent initial evaluation and/or their proxies for their clinical records to be used in this study. All patient records and information were anonymized and de-identified prior to the analysis.

\subsection{Multidimensional Prognostic Index (MPI)}

The MPI, recorded in the first $48 \mathrm{~h}$ after admission, was calculated from information obtained through a standard CGA that considered the following eight different domains [16,17]:

1 Functional status as evaluated by Katz's Activities of Daily Living (ADL) index [18], which defines the level of dependence/independence in six daily personal care activities (bathing, toileting, feeding, dressing, urine and bowel continence and transferring (in and out of bed or chair);

2 Independence in the Lawton's Instrumental Activities of Daily Living (IADL) [19] which assesses independence in eight activities that are more cognitively and physically demanding than ADL, i.e. managing finances, using telephone, taking medications, hopping, using transportation, preparing meals, doing housework and washing;

3 Cognitive status through the Short Portable Mental Status Questionnaire (SPMSQ) [20], a ten item questionnaire investigating orientation, memory, attention, calculation, and language; validated versions were used in each local language.

4 Co-morbidity was examined using the Cumulative Illness Rating Scale (CIRS). ${ }^{20}$ The CIRS uses a 5-point ordinal scale (score 1-5) to estimate the severity of pathology in each of 13 systems, including cardiac, vascular, respiratory, eye-ear-nose-throat, upper and lower gastrointestinal, hepatic, renal, genitourinary, musculoskeletal, skin disorder, nervous system, endocrine-metabolic and psychiatric behavioural disorders. Based on the ratings, the Comorbidity Index (CIRS-CI) score, which reflects the number of concomitant diseases, was derived from the total number of categories in which moderate or severe levels (grade from 3 to 5) of disease were identified (range from 0 to 13). Comorbidities, at hospital admission, were reported using the International Classification of Disease, 10th version.

5 Nutritional status was investigated with the Mini Nutritional Assessment Short Form (MNA-SF) [21], which includes information on: a) anthropometric measures (body mass index, BMI; weight loss); b) neuropsychological problems and recent psychological stress; c) mobility; d) decline in food intake.

6 Risk of developing pressure sores was evaluated through the Exton Smith Scale (ESS), a five items questionnaire determining physical and mental condition, activity, mobility and incontinence [22].

7 Medication use was defined according to the Anatomical Therapeutics Chemical (ATC) classification code system and the number of drugs used by patients at admission was recorded. Patients were defined as drug users if they took a medication included in the ATC classification at the moment of hospital admission.

8 Cohabitation status included living alone, in an institution, or with family members.

For each domain, a tripartite hierarchy was used, i.e. $0=$ no problems, $0.5=$ minor problems, and $1=$ major problems, based on conventional cut-off points derived from the literature for each item. The sum of the calculated scores from the eight domains was divided by 8 to obtain a final MPI risk score ranging from $0=$ no risk to $1=$ higher risk of mortality [16]. The MPI assessment requires between 15-25 minutes and the results can be automatically generated using the MPI calculator software downloaded from the www.mpiage.eu website. 


\subsection{Main outcomes}

All the participants were followed for 12 months from hospital discharge. Mortality was consequently categorized as in-hospital or post discharge. Vital status was assessed by consulting the Registry Offices of the cities in which the patients were resident.

Overall mortality (in-hospital and one-year, at home death) was considered as primary outcome of our paper, whilst institutionalization (in terms of long-term care or nursing home access), re-hospitalization (within one year from discharge) and access to homecare services were considered as secondary outcomes. The secondary outcomes were investigated using administrative data and phone interviews.

\subsection{Statistical analysis}

A sample size of 1000 participants was deemed sufficient, assuming a fixed Type I error alpha of $5 \%$ and a statistical power of $80 \%$, to detect an increase in mortality at one-year equivalent to a risk of at least $36 \%$ per those participants classified as MPI 2 and to a hazard ratio of at least $37 \%$ for those participants classified as MPI 3, set the MPI class 1 as the reference. It was taken a one-year mortality for the reference class equal to $10 \%$ [23].

General characteristics were reported as frequencies (percentages) and mean \pm standard deviation (SD), for categorical and continuous variables, respectively. Mortality incidence rates were computed as the number of deaths per person-year, by sex, and compared using the logrank test. Comparisons between men and women were performed using the chi-square test and the Student's $t$-test for independent groups, for categorical and continuous variables, respectively.

Logistic binary regression analysis was run, taking as exposure sex and overall, in-hospital and at home, mortality as outcomes. Similar analyses were run for institutionalization and re-hospitalization as outcomes. The strength of the association between sex and primary/ secondary outcomes were reported as odds ratios (ORs) with their 95\% confidence intervals (CIs), adjusted for age, MPI at admission, center and primary diagnosis. The primary diagnosis was recorded through medical information at the admission and classified using the ICD10.

Two-sided alternatives with a significance level $\alpha=0.05$ were considered for all the tests. STATA 12 (StataCorp, Stata Statistical Software: Release 12, College Station, TX, USA) software was used.

\section{Results}

\subsection{Characteristics of the study population}

The study population included 1140 hospitalized patients (mean age $84.2 \pm 7.4$ years), with 694 women $(=60.9 \%)$ and 446 men ( $=39.1 \%)$.

Table 1 shows the characteristics of the participants by sex. Women were significantly older $(\mathrm{p}<0.0001)$ and had a significant higher mean MPI value on admission $(\mathrm{p}=0.029)$ than men. Consequently, men were categorized more frequently than women in mild risk of mortality (MPI 1) (19.1 vs. $12.1 \%$; p = 0.005). Regarding the single items of the MPI, women reported significant lower CIRS scores than men ( $\mathrm{p}<0.001$ ), and they lived more frequently alone than men before hospitalization (40.8 vs. 20.0\%, p < 0.001) (Table 1).

As regards the diagnoses at hospital discharge, men were hospitalized more frequently than women for cancer $(7.7 \%$ vs. $3.2 \%$; $\mathrm{p}=0.002)$ or for respiratory diseases $(19.3 \%$ vs. $13.3 \%$; $p=0.009)$, while women were hospitalized more frequently than men for fractures and falls/traumas (16.4 vs. 8.1\%; p < 0.001) (Table 1).

\subsection{Sex and mortality}

Table 2 shows the association between sex and mortality. The mortality rate, by sex, is graphically reported in Fig. 1. In multivariate analysis adjusting for age, MPI at admission, centre and diagnosis at discharge, men died more frequently than women $(\mathrm{OR}=2.04$; 95\%CI: 1.50-2.79; $\mathrm{p}<0.001)$. These findings were confirmed when considering in-hospital mortality $(\mathrm{OR}=2.26$; 95\%CI: 1.27-4.01; $\mathrm{p}=0.005)$ or one-year mortality after discharge (OR $=2.11 ; 95 \% \mathrm{CI}$ : 1.49-3.00; $\mathrm{p}<0.001$ ) as separate outcomes (Table 2).

\subsection{Sex and risk of institutionalization and re-hospitalization}

Overall, after adjusting for potential confounders, men were less frequently institutionalized than women, $(\mathrm{OR}=0.55$; 95\%CI: $0.34-0.91 ; \mathrm{p}=0.02)$, but they were more frequently re-hospitalized $(\mathrm{OR}=1.42 ; 95 \% \mathrm{CI}$ : 1.06-1.91; $\mathrm{p}=0.02)$, during follow-up (Table 3).

\section{Discussion}

In this prospective observational study of a large international cohort of hospitalized older individuals, we found that women, albeit more frail and older, reported a significant lower mortality rate than men. Moreover, men were less frequently institutionalized than women, but they were more frequently re-hospitalized during one year of follow-up. Overall, these findings extend the 'male-female health-survival paradox' [24], i.e. that women, even if they are more frail, are less likely to die than men, to the acute care setting.

Notably, frailty in our study was assessed using the MPI. This CGAbased tool exhibits the highest validity, reliability and feasibility, when compared to other scoring systems, in the identification and risk stratification of frail older patients in hospital [25]. The MPI is an accurate predictor of short- and long-term mortality and length of hospital stay [26] as well as of the risk of institutionalization, re-hospitalization and access to homecare services in hospitalized older persons [27].

Previous studies have investigated sex differences in frailty in older people. In a large European study, the years lived free from frailty or physical limitations were significantly less for women than men, probably due to socio-economic as well as biological factors [28]. Indeed, also genetic sex differences (i.e. $\varepsilon 2$ allele prevalence) could partially explain the differences in healthy aging, frailty and mortality observed between men and women, as reported in previous studies [29]. Although there are known differences in which men and women self-reported their health [30], some authors suggest that the greater prevalence of frailty in older women may represent a male 'fitness-frailty pleiotropy' and/or a female 'fertility-frailty pleiotropy', finally resulting in men with lower physiological reserves in old age [31], and consequently a higher risk of death. In line with this hypothesis, in a recent randomized placebo-controlled trial, amino acid supplementation significantly improved frailty status (as assessed by the MPI) in hospitalized older male, but not female, patients [32].

Other authors suggested that socio-economic issues could be more important than biological mechanisms in explaining our findings. A study made in the SHARE project showed, in fact, the presence of various sources of social differences not only in middle-aged, but also in older people, that may affect more women than men [28]. Our work supports this hypothesis, since women lived more frequently alone than men (40.8\% vs. $20.0 \%$ ), even if no significant differences in basic and instrumental daily living activities were reported. In a large Italian study of hospitalized older people, living alone was associated with a higher risk of non-home discharge, whereas living alone was not significantly associated with in-hospital mortality [33], in line with our findings. Another explanation could be that women reported less comorbidity severity than men, as shown by lower CIRS-SI scores, and were hospitalized for reasons (e.g. fractures and falls) that are probably less life-threatening than those reported in men (e.g. cancer). Taking together, our work furtherly confirmed the sex differences between men and women in mortality, extending our knowledge regarding this topic in hospital setting.

Our study also reports important differences in secondary outcomes 
Table 1

General characteristics of older patients at baseline according to sex.

\begin{tabular}{|c|c|c|c|c|c|}
\hline & & $\begin{array}{l}\text { ALL patients } \\
1140\end{array}$ & $\begin{array}{l}\text { FEMALE } \\
694(60.9 \%)\end{array}$ & $\begin{array}{l}\text { MALE } \\
446(39.1 \%)\end{array}$ & $p^{*}$ \\
\hline \multicolumn{2}{|l|}{ Length of stay* (mean, SD) } & $14.1(11.8)$ & $14.1(11.7)$ & $14.1(11.9)$ & 0.915 \\
\hline \multicolumn{2}{|l|}{ Age (mean, SD) } & $84.2(7.4)$ & $84.9(7.2)$ & $83.1(7.4)$ & $<0.001$ \\
\hline \multicolumn{2}{|l|}{ BMI, kg/m² (mean, SD) } & $25.5(5.1)$ & $25.5(5.2)$ & $25.4(4.9)$ & 0.788 \\
\hline \multicolumn{2}{|l|}{ Height, cm (mean, SD) } & $163.2(9.5)$ & $158.5(7.3)$ & $170.1(8.0)$ & $<0.001$ \\
\hline \multicolumn{2}{|l|}{ Weight, kg (mean, SD) } & $68.0(15.2)$ & $64.1(13.6)$ & $73.8(15.5)$ & $<0.001$ \\
\hline \multicolumn{2}{|l|}{ Years of education (mean, SD) } & $6.8(4.4)$ & $6.6(4.0)$ & $7.1(4.9)$ & 0.111 \\
\hline \multicolumn{2}{|l|}{ MPI at Admission (mean, SD) } & $0.58(0.20)$ & $0.59(0.20)$ & $0.57(0.21)$ & 0.029 \\
\hline \multirow[t]{3}{*}{ MPI at Admission (class) } & mild & $169(14.8 \%)$ & $84(12.1 \%)$ & $85(19.1 \%)$ & 0.005 \\
\hline & moderate & $502(44.0 \%)$ & $319(46.0 \%)$ & $183(41.0 \%)$ & \\
\hline & severe & $469(41.1 \%)$ & $291(41.9 \%)$ & $178(39.9 \%)$ & \\
\hline \multicolumn{2}{|c|}{ Activities of Daily Living (mean, SD) } & $2.8(2.3)$ & $2.7(2.3)$ & $2.9(2.3)$ & 0.348 \\
\hline \multicolumn{2}{|c|}{ Instrumental Activities of Daily Living (mean, SD) } & $2.7(2.6)$ & $2.7(2.5)$ & $2.6(2.6)$ & 0.545 \\
\hline \multicolumn{2}{|c|}{ Short Portable Mental Status Questionnaire (mean, SD) } & $4.1(3.5)$ & $4.2(3.5)$ & $4.0(3.5)$ & 0.507 \\
\hline \multicolumn{2}{|c|}{ Mini Nutritional Assessment Short Form (mean, SD) } & $8.6(3.3)$ & $8.5(3.3)$ & $8.7(3.3)$ & 0.504 \\
\hline \multicolumn{2}{|c|}{ Exton-Smith scale (mean, SD) } & $14.1(3.7)$ & $14.1(3.6)$ & $14.1(3.8)$ & 0.976 \\
\hline \multicolumn{2}{|c|}{ Comorbidity Index Rating Scale (mean, SD) } & $2.1(0.4)$ & $2.1(0.4)$ & $2.2(0.4)$ & $<0.001$ \\
\hline \multicolumn{2}{|c|}{ Number of medications (mean, SD) } & $7.1(3.4)$ & $7.0(3.2)$ & $7.3(3.5)$ & 0.239 \\
\hline \multirow[t]{3}{*}{ Cohabitation status (n, \%) } & Alone & $372(32.7 \%)$ & $283(40.8 \%)$ & $89(20.0 \%)$ & $<0.001$ \\
\hline & with family or private care & $623(54.6 \%)$ & $325(46.9 \%)$ & $298(66.8 \%)$ & \\
\hline & in institution/homecare & $144(12.6 \%)$ & $85(12.3 \%)$ & $59(13.2 \%)$ & \\
\hline \multirow[t]{12}{*}{ Main diagnosis** by ICD10 (n, \%): } & AB- Infectious & $87(7.8 \%)$ & $52(7.6 \%)$ & $35(8.1 \%)$ & 0.819 \\
\hline & CD- Neoplasms & $55(4.9 \%)$ & $22(3.2 \%)$ & $33(7.7 \%)$ & 0.002 \\
\hline & D5- Blood & $34(3.1 \%)$ & $17(2.5 \%)$ & $17(3.9 \%)$ & 0.210 \\
\hline & E - Endocrine, nutritional and metabolism & $41(3.7 \%)$ & $29(4.2 \%)$ & $12(2.8 \%)$ & 0.253 \\
\hline & FG - Nervous system and behavioural & $81(7.3 \%)$ & $53(7.7 \%)$ & $28(6.5 \%)$ & 0.478 \\
\hline & HLT - Skin. ear and others & $25(2.2 \%)$ & $15(2.2 \%)$ & $10(2.3 \%)$ & 1.000 \\
\hline & I - Circulatory system & $260(23.3 \%)$ & $151(22.0 \%)$ & $109(25.3 \%)$ & 0.217 \\
\hline & J - Respiratory system & $174(15.6 \%)$ & $91(13.3 \%)$ & $83(19.3 \%)$ & 0.009 \\
\hline & $\mathrm{K}$ - Digestive system & $103(9.2 \%)$ & $73(10.7 \%)$ & $30(7.0 \%)$ & 0.043 \\
\hline & M - Musculoskeletal and connective & $73(6.5 \%)$ & $50(7.3 \%)$ & $23(5.3 \%)$ & 0.215 \\
\hline & $\mathrm{N}$ - Genitourinary system & $36(3.2 \%)$ & $20(2.9 \%)$ & $16(3.7 \%)$ & 0.489 \\
\hline & RS - Fractures, tendency to fall, edema & $147(13.2 \%)$ & $112(16.4 \%)$ & $35(8.1 \%)$ & $<0.001$ \\
\hline
\end{tabular}

Abbreviations: SD: standard deviation; ICD: International Classification Disease.

*: length of stay did not include the period in the Emergency Department; **Diagnosis was missing in 24 patients.

between men and women. Women, in fact, were admitted to nursing homes more frequently, but were also re-hospitalized less frequently than men. It is possible that women admitted to a nursing home received better care, hence reduced risk of re-hospitalization, than men returning at their home [34]. The higher social isolation of women, living more frequently alone before hospitalization, might also explain their more frequent admission to a nursing home after discharge. We believe that this is an important and novel finding of our work, suggesting public health interventions in order to strictly monitor women after hospital discharge that seem more vulnerable in terms of nursing home placements.

The findings of our study should be interpreted within its limitations. First, the follow-up of these patients was limited to one year, therefore we cannot rule out different sex-related patterns in negative outcomes with longer follow-up. Second, frailty is not necessarily a progressive syndrome, and transitions between frailty categories over time frequently occur at an individual level, potentially affecting our results. Third, we assessed only MPI as measure of frailty in this cohort. In a previous research, in a similar cohort, all frailty instruments (based on Study of Osteoporotic Fractures criteria, based on the cumulative deficits model or based on a comprehensive geriatric assessment) were

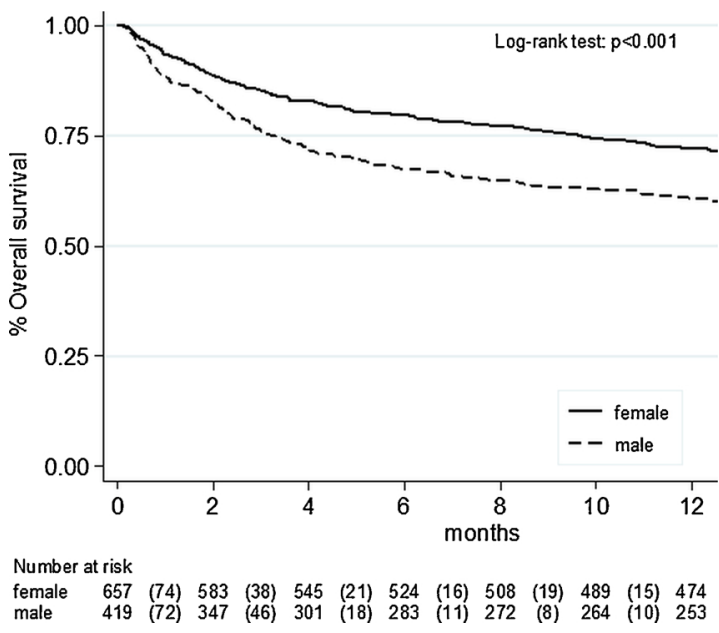

Fig. 1. Differences in mortality between male and female sex in hospitalized older people.

Table 2

Association between sex and mortality in hospitalized older patients.

\begin{tabular}{|c|c|c|c|c|c|}
\hline Outcome & ALL patients & FEMALE & MALE & $\mathrm{OR}^{2} \mathrm{M}$ vs $\mathrm{F}(95 \% \mathrm{CI})$ & $p$ \\
\hline In-hospital mortality & $75(6.7 \%)$ & $35(5.0 \%)$ & $40(9.0 \%)$ & $2.26(1.27-4.01)$ & 0.005 \\
\hline One-year post-discharge mortality ${ }^{1}$ & $283(28.2 \%)$ & $154(24.7 \%)$ & $129(33.9 \%)$ & $2.11(1.49-3.00)$ & $<0.001$ \\
\hline
\end{tabular}

Notes: (1) 61 drops out; (2) ORs were adjusted for age, MPI, Centre and Diagnosis.

Abbreviations: CI: confidence intervals; OR: odds ratio; MPI: Multidimensional Prognostic Index. 
Table 3

Association between sex and secondary outcomes in hospitalized older people.

\begin{tabular}{|c|c|c|c|c|c|}
\hline Outcome & ALL patients ${ }^{1}$ & FEMALE & MALE & $\mathrm{OR}^{3} \mathrm{M}$ vs $\mathrm{F}(95 \% \mathrm{CI})$ & $p$ \\
\hline Instituzionalization $^{2}$ & $149(17.0 \%)$ & $108(19.8 \%)$ & $41(12.4 \%)$ & $0.55(0.34-0.91)$ & 0.020 \\
\hline Re-hospitalization & $572(57.0 \%)$ & 337 (54.1\%) & $235(61.7 \%)$ & $1.42(1.06-1.91)$ & 0.020 \\
\hline
\end{tabular}

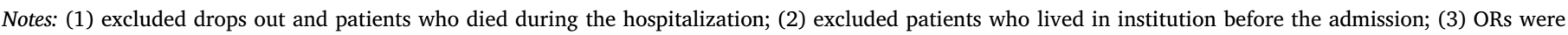
adjusted for age, MPI, Centre and Diagnosis at Discharge.

Abbreviations: CI: confidence intervals; OR: odds ratio; MPI: Multidimensional Prognostic Index.

significantly associated with short- and long-term all-cause mortality, but MPI showed a significant higher predictive power than other frailty instruments in hospitalized older patients. Finally, the causes of death were not included, but this could be a further area of research.

In conclusion, in this cohort of hospitalized older adults, men died more frequently than women over one year of follow-up. However, women were admitted more frequently than men in nursing home and experienced a lower risk of re-hospitalization, suggesting important differences between older men and women.

\section{Contributors}

Nicola Veronese contributed to study concept and design, and preparation of the manuscript

Giacomo Siri contributed to analysis and interpretation of data.

Alberto Cella contributed to acquisition of subjects and/or data.

Julia Daragjati contributed to acquisition of subjects and/or data.

Alfonso J. Cruz-Jentoft contributed to preparation of manuscript.

Maria Cristina Polidori contributed to revision of the manuscript.

Francesco Mattace-Raso contributed to preparation of the manuscript.

Marc Paccalin contributed to preparation of the manuscript.

Eva Topinkova contributed to preparation of the manuscript.

Antonio Greco contributed to preparation of the manuscript.

Arduino A Mangoni contributed to revision of the manuscript.

Stefania Maggi contributed to revision of the manuscript.

Luigi Ferrucci contributed to revision of the manuscript.

Alberto Pilotto contributed to study concept and design, preparation of the manuscript.

\section{Funding}

This work was supported by the MPI_AGE European project cofunded by the Consumers, Health, Agriculture, and Food Executive Agency (CHAFEA) in the frame of the European Innovation Partnership on Active and Healthy Ageing Second Health Programme 2008-2013.

The sponsor had no role in the design, methods, subject recruitment, data collections, analysis and preparation of the paper.

\section{Ethical approval}

The ethics committees of each center approved this observational study. Informed consent was given by participants who underwent initial evaluation and/or their proxies for their clinical records to be used in this study. All patient records and information were anonymized and de-identified prior to the analysis.

\section{Provenance and peer review}

This article has undergone peer review.

\section{Research data (data sharing and collaboration)}

There are no linked research data sets for this paper. Data will be made available on reasonable request to the corresponding author.

\section{Declaration of Competing Interest}

The authors declare that they have no conflict of interest.

\section{References}

[1] S.N. Austad, A. Bartke, Sex differences in longevity and in responses to anti-aging interventions: a mini-review, Gerontology 62 (1) (2016) 40-46.

[2] E. Gordon, N. Peel, M. Samanta, O. Theou, S. Howlett, R. Hubbard, Sex differences in frailty: a systematic review and meta-analysis, Exp. Gerontol. 89 (2017) 30-40.

[3] R.M. Collard, H. Boter, R.A. Schoevers, R.C. Oude Voshaar, Prevalence of frailty in community-dwelling older persons: a systematic review, J. Am. Geriatr. Soc. 60 (8) (2012) 1487-1492.

[4] T. Shamliyan, K.M. Talley, R. Ramakrishnan, R.L. Kane, Association of frailty with survival: a systematic literature review, Ageing Res. Rev. 12 (2) (2013) 719-736.

[5] A. Oksuzyan, J. Gumà, G. Doblhammer, Sex Differences in Health and Survival, a Demographic Perspective on Gender, Family and Health in Europe, Springer, Cham, 2018, pp. 65-100.

[6] A. Mitnitski, X. Song, I. Skoog, G. Broe, J.L. Cox, E. Grunfeld, K. Rockwood, Relative fitness and frailty of elderly men and women in developed countries and their relationship with mortality, J. Am. Geriatr. Soc. 53 (12) (2005) 2184-2189.

[7] I.-M. Berges, J.E. Graham, G.V. Ostir, K.S. Markides, K.J. Ottenbacher, Sex differences in mortality among older frail Mexican Americans, J. Womens Health 18 (10) (2009) 1647-1651.

[8] K.-U. Saum, A.K. Dieffenbach, H. Müller, B. Holleczek, K. Hauer, H. Brenner, Frailty prevalence and 10-year survival in community-dwelling older adults: results from the ESTHER cohort study, Eur. J. Epidemiol. 29 (3) (2014) 171-179.

[9] M.T.E. Puts, P. Lips, D.J.H. Deeg, Sex differences in the risk of frailty for mortality independent of disability and chronic diseases, J. Am. Geriatr. Soc. 53 (1) (2005) $40-47$.

[10] A.M. Kulminski, I.V. Culminskaya, S.V. Ukraintseva, K.G. Arbeev, K.C. Land, A.I. Yashin, Sex-specific health deterioration and mortality: the morbidity-mortality paradox over age and time, Exp. Gerontol. 43 (12) (2008) 1052-1057.

[11] J. Kulmala, I. Nykänen, S. Hartikainen, Frailty as a predictor of all-cause mortality in older men and women, Geriatr. Gerontol. Int. 14 (4) (2014) 899-905.

[12] J. Tropea, D. LoGiudice, D. Liew, A. Gorelik, C. Brand, Poorer outcomes and greater healthcare costs for hospitalised older people with dementia and delirium: a retrospective cohort study, Int. J. Geriatr. Psychiatry 32 (5) (2017) 539-547.

[13] E. Chong, E. Ho, J. Baldevarona-Llego, M. Chan, L. Wu, L. Tay, Y.Y. Ding, W.S. Lim, Frailty in hospitalized older adults: comparing different frailty measures in predicting short-and long-term patient outcomes, J. Am. Med. Dir. Assoc. 19 (5) (2018) 450-457. e3.

[14] Q. Hao, L. Zhou, B. Dong, M. Yang, B. Dong, Y. Weil, The role of frailty in predicting mortality and readmission in older adults in acute care wards: a prospective study, Sci. Rep. 9 (1) (2019) 1207.

[15] R.B. Parker, T. Larkin, J. Cockburn, Gender bias in medical images affects students implicit but not explicit gender attitudes, AERA Open 4 (3) (2018) 2332858418798832.

[16] A. Pilotto, L. Ferrucci, M. Franceschi, L.P. D'Ambrosio, C. Scarcelli, L. Cascavilla, F. Paris, G. Placentino, D. Seripa, B. Dallapiccola, G. Leandro, Development and validation of a multidimensional prognostic index for one-year mortality from comprehensive geriatric assessment in hospitalized older patients, Rejuvenation Res. 11 (1) (2008) 151-161.

[17] A. Pilotto, F. Rengo, N. Marchionni, D. Sancarlo, A. Fontana, F. Panza, L. Ferrucci, Comparing the prognostic accuracy for all-cause mortality of frailty instruments: a multicentre 1-year follow-up in hospitalized older patients, PLoS One 7 (1) (2012) e29090.

[18] S. Katz, T.D. Downs, H.R. Cash, R.C. Grotz, Progress in development of the index of ADL, Gerontologist 10 (1) (1970) 20-30.

[19] M.P. Lawton, E.M. Brody, Assessment of older people: self-maintaining and instrumental activities of daily living, Gerontologist 9 (3) (1969) 179-186.

[20] E. Pfeiffer, A Short Portable Mental Status Questionnaire (SPMSQ), J. Am. Geriatr. Soc. 23 (10) (1975) 1975.

[21] D. Sancarlo, G. D’Onofrio, M. Franceschi, C. Scarcelli, V. Niro, F. Addante, M. Copetti, L. Ferrucci, L. Fontana, A. Pilotto, Validation of a ModifiedMultidimensional Prognostic Index (m-MPI) including the Mini Nutritional Assessment Short-Form (MNA-SF) for the prediction of one-year mortality in hospitalized elderly patients, J. Nutr. Health Aging 15 (3) (2011) 169-173.

[22] M.R. Bliss, R. McLaren, A.N. Exton-Smith, Mattresses for preventing pressure sores in geriatric patients, Mon. Bull. Minist. Health Public Health Lab. Serv. 25 (1966) $238-268$. 
[23] A. Pilotto, F. Panza, L. Ferrucci, A multidimensional prognostic index in common conditions leading to death in older patients, Arch. Intern. Med. 172 (7) (2012) 594 discussion 594-5.

[24] A. Oksuzyan, I. Petersen, H. Stovring, P. Bingley, J.W. Vaupel, K. Christensen, The male-female health-survival paradox: a survey and register study of the impact of sex-specific selection and information bias, Ann. Epidemiol. 19 (7) (2009) 504-511.

[25] R. Warnier, E. Van Rossum, E. Van Velthuijsen, W. Mulder, J. Schols, G. Kempen, Validity, reliability and feasibility of tools to identify frail older patients in inpatient hospital care: a systematic review, J. Nutr. Health Aging 20 (2) (2016) 218-230.

[26] S. Volpato, S. Bazzano, A. Fontana, L. Ferrucci, A. Pilotto, Multidimensional Prognostic Index predicts mortality and length of stay during hospitalization in the older patients: a multicenter prospective study, J. Gerontol. A Biol. Sci. Med. Sci. 70 (3) (2015) 325-331.

[27] A. Pilotto, N. Veronese, J. Daragjati, A.J. Cruz-Jentoft, M.C. Polidori, F. MattaceRaso, M. Paccalin, E. Topinkova, G. Siri, A. Greco, A.A. Mangoni, S. Maggi, L. Ferrucci, Using the multidimensional prognostic index to predict clinical outcomes of hospitalized older persons: a prospective, multicentre, international study, J. Gerontol. A Biol. Sci. Med. Sci. (2018).

[28] R. Romero-Ortuno, T. Fouweather, C. Jagger, Cross-national disparities in sex differences in life expectancy with and without frailty, Age Ageing 43 (2) (2013) 222-228.

[29] D. Seripa, M. Franceschi, M.G. Matera, F. Panza, P.G. Kehoe, C. Gravina, G. Orsitto,
V. Solfrizzi, G. Di Minno, B. Dallapiccola, Sex differences in the association of apolipoprotein E and angiotensin-converting enzyme gene polymorphisms with healthy aging and longevity: a population-based study from Southern Italy, J. Gerontol. A Biol. Sci. Med. Sci. 61 (9) (2006) 918-923.

[30] N. Spiers, C. Jagger, M. Clarke, A. Arthur, Are gender differences in the relationship between self-rated health and mortality enduring? Results from three birth cohorts in Melton Mowbray, United Kingdom, Gerontologist 43 (3) (2003) 406-411.

[31] R.E. Hubbard, K. Rockwood, Frailty in older women, Maturitas 69 (3) (2011) 203-207.

[32] S. Volpato, R. Custureri, M. Puntoni, L. Bianchi, J. Daragjati, S. Garaboldi, M. Simonato, A. Greco, E. Rizzo, P. Dal Santo, Effects of oral amino acid supplementation on Multidimensional Prognostic Index in hospitalized older patients: a multicenter randomized, double-blind, placebo-controlled pilot study, Clin. Interv. Aging 13 (2018) 633.

[33] P. Agosti, M. Tettamanti, F. Vella, P. Suppressa, L. Pasina, C. Franchi, A. Nobili, P. Mannucci, C. Sabbà, R. Investigators, Living alone as an independent predictor of prolonged length of hospital stay and non-home discharge in older patients, Eur. J. Intern. Med. (2018).

[34] M.P. Scapinello, A. Posocco, I. De Ronch, F. Castrogiovanni, G. Lollo, G. Sergi, I. Tomaselli, L. Tonon, M. Solmi, S. Traversa, V. Zambianco, N. Veronese, Predictors of emergency department referral in patients using out-of-hours primary care services, Health Policy (Amsterdam, Netherlands) 120 (9) (2016) 1001-1007. 